\title{
On heat transfer at microscale with implications for microactuator design
}

\author{
Ozgur Ozsun $^{1}$, B Erdem Alaca ${ }^{1,4}$, Arda D Yalcinkaya ${ }^{2}$, \\ Mehmet Yilmaz $^{1,5}$, Michalis Zervas ${ }^{3}$ and Yusuf Leblebici ${ }^{3}$ \\ ${ }^{1}$ Department of Mechanical Engineering, Koc University, Rumeli Feneri Yolu, 34450 Sariyer, Istanbul, \\ Turkey \\ ${ }^{2}$ Department of Electrical and Electronics Engineering, Bogazici University, 34342 Bebek, Istanbul, \\ Turkey \\ ${ }^{3}$ Microelectronic Systems Laboratory, EPFL, CH-1015 Lausanne, Switzerland \\ E-mail: ealaca@ku.edu.tr
}

Received 8 August 2008, in final form 15 February 2009

Published 20 March 2009

Online at stacks.iop.org/JMM/19/045020

\begin{abstract}
The dominance of conduction and the negligible effect of gravity, and hence free convection, are verified in the case of microscale heat sources surrounded by air at atmospheric pressure. A list of temperature-dependent heat transfer coefficients is provided. In contrast to previous approaches based on free convection, supplied coefficients converge with increasing temperature. Instead of creating a new external function for the definition of boundary conditions via conductive heat transfer, convective thin film coefficients already embedded in commercial finite element software are utilized under a constant heat flux condition. This facilitates direct implementation of coefficients, i.e. the list supplied in this work can directly be plugged into commercial software. Finally, the following four-step methodology is proposed for modeling: (i) determination of the thermal time constant of a specific microactuator, (ii) determination of the boundary layer size corresponding to this time constant, (iii) extraction of the appropriate heat transfer coefficients from a list provided and (iv) application of these coefficients as boundary conditions in thermomechanical finite element simulations. An experimental procedure is established for the determination of the thermal time constant, the first step of the proposed methodology. Based on conduction, the proposed method provides a physically sound solution to heat transfer issues encountered in the modeling of thermal microactuators.
\end{abstract}

(Some figures in this article are in colour only in the electronic version)

\section{Introduction}

Thermal actuation is preferred in applications where large deflections are required at low to moderate loads. In contrast to electrostatic or piezoelectric actuation, considerable deflections can be achieved at low voltages. Two widely used thermal actuators are the Guckel [1] thermal actuator for in-plane actuation and the bimorph [2] thermal actuator for out-of-plane motion. The operation of the Guckel actuator is based on the non-uniform expansion of thin and wide arms,

4 Author to whom any correspondence should be addressed.

5 Present address: Department of Mechanical Engineering, Columbia University, 500 West 120th Street, New York, NY 10027, USA. whereas a bimorph actuator utilizes two layers of materials with different coefficients of thermal expansion.

Microgrippers constitute a good example for the practical use of thermal actuators. Such devices can carry out tasks including pick-and-place assembly and soldering or welding along with the capability of sensory feedback [3]. An interesting application of such pick-and-place assembly is the handling of carbon nanotubes with an electrothermal gripper in a scanning electron microscope [4]. Grippers can as well be used in aqueous media for biomedical applications including cell manipulation [5].

In the modeling of thermal actuators, the electro/photothermal problem and the thermo-mechanical problem are 
generally addressed in a sequential manner. The first problem is concerned with the computation of the temperature distribution on the device. To do this, knowledge regarding the dominant modes of heat transfer is necessary for the definition of appropriate boundary conditions on device surfaces where energy is exchanged with the surrounding medium. This is where a variety of assumptions are adopted in the literature. This issue will be discussed in detail in the next section. The second, i.e. thermo-mechanical, part of the solution procedure is then employed to compute displacements resulting from the corresponding temperature distribution.

The main purpose of this work is to clearly highlight the negligible effect of free convection compared to conduction at microscale. The negligence toward this fact present in the modeling literature is more than occasional. What is missing is a methodology that would allow the assignment of conductive heat flux as a boundary condition. In this work, a set of temperature-dependent conductive heat transfer coefficients will be defined that can be embedded into commercial finite element software. The manuscript is organized in the following way: after a brief introduction of basic concepts, two models for heat transfer in solid and fluid media will be constructed to compare and contrast micro- and macroscales in terms of dominant modes of heat transfer. Based on this perspective, a literature review will follow, where the necessity of the present work will be highlighted. Then a methodology will be introduced for computing temperature-dependent heat transfer coefficients. Finally, the methodology will be applied to a specific bimorph microactuator, and the experimental results of the voltage-deflection characteristics of the actuator will be compared with finite element simulations utilizing the new set of conductive heat transfer coefficients.

\section{Theory}

The dominant mode of heat transfer in a fluid medium depends on the length scale. For example, if one considers heat transfer from a solid surface to surrounding air at macroscale, free convection would turn out to be the dominant factor. However, at microscale, free convection is negligible when compared to conduction. Hence, in the absence of forced convection, conduction should be treated as the only mode of heat transfer for the modeling of microscale thermal actuators. The widespread use of free convection in the modeling literature is in contradiction with this fact. To shed light on this issue, basic concepts of heat transfer will be introduced first. This will be followed by a finite element study of heat transfer in a chosen geometry at different length scales. The section will be concluded with an evaluation of different approaches to the modeling of microscale thermal actuators.

\subsection{Basic concepts}

The transfer of heat can take place in different modes. Conduction is the transfer of energy based purely on molecular activity. In a fluid, conduction occurs through random motion of molecules. For a solid, this would translate into lattice waves due to molecular motion in addition to the motion of free electrons in the case of conductors. Convection takes place in the presence of bulk motion of a fluid. It is especially important in the case of energy transfer across a solid/fluid interface. Finally, energy can be emitted as thermal radiation. In this work, the interplay between conduction and convection will be considered with special emphasis placed on heat transfer across a solid/fluid interface.

There are two basic types of convection, namely free-or natural-convection and forced convection. Forced convection requires an external means to set fluid in motion. Free convection is driven by buoyancy forces representing the gravitational effect on the fluid density gradient which is due to the presence of a temperature gradient. The criterion that determines which component of convection is dominant is provided by the non-dimensional Grashof number, $G r$. $G r$ represents the ratio of buoyancy forces to viscous forces and is defined in table 1 along with other important nondimensional numbers such as Reynolds number, $R e$. Since $R e$ represents the ratio of inertial to viscous forces, the condition of $\left(G r / R e^{2} \gg 1\right)$ corresponds to the dominance of free convection over forced convection. Typical values of $\mathrm{Gr}$ for microstructures are on the order of $10^{-8}$ to $10^{-2}$.

Similarly, Prandtl number, $\mathrm{Pr}$, can be considered as the ratio of the momentum and thermal diffusivities. For small $\mathrm{Pr}$, heat diffuses faster than momentum, i.e. thermal boundary layer is shorter than velocity boundary layer. $\operatorname{Pr}$ for air at $300 \mathrm{~K}$ is 0.707 and decreases as the temperature increases such that its value is 0.536 at $3000 \mathrm{~K}$.

Another non-dimensional number that is used interchangeably with $G r$ is the Rayleigh number, $R a$, which is the product of $G r$ and $P r$. Ra serves as a criterion in deciding the type of heat transfer in fluids. If $R a$ is smaller than a certain critical value for that fluid, the dominant heat transfer mechanism is conduction and if that critical value is exceeded, then convection takes over. Typical values of $R a$ for microstructures are on the order of $10^{-9}$ to $10^{-3}$.

Finally, the non-dimensional Nusselt number, $N u$, is defined as the dimensionless temperature gradient at the surface. It is treated as a measure of the convective heat transfer occurring at the surface. The empirical correlations between $\mathrm{Nu}$ and $\mathrm{Ra}$ for flat plates are given in the form of

$$
N u=C R a^{n / m}
$$

where $C, n$ and $m$ are constants with values depending on the range of $R a$ among other factors [6]. The most important implication of equation (1) is the computation of temperaturedependent convection coefficients, $h$. These can be extracted by combining equation (1) with the definitions of $\mathrm{Nu}$ and $\mathrm{Ra}$ provided in table 1 . The critical point associated with this procedure is that correct values for the constants $n, m$ and $C$ should be taken with consideration of the $R a$ range of the convective surface. This issue will be highlighted later in the literature review.

\subsection{Scale dependence of heat transfer modes}

In order to compare micro and macroscales in terms of dominant modes of heat transfer, a set of simulations is 
Table 1. A list of important non-dimensional numbers (compiled with data from [6]).

Non-dimensional

number

Reynolds number $\quad R e=\frac{V L}{v}$

Grashof number

$$
G r=\frac{g \beta\left(T_{s}-T_{\infty}\right) L^{3}}{v^{2}}
$$

Prandtl number $\quad \operatorname{Pr}=\frac{v}{\alpha}$

Rayleigh number

$$
R a=G r \operatorname{Pr}=\frac{g \beta\left(T_{s}-T_{\infty}\right) L^{3}}{\nu \alpha}
$$

Product of $\mathrm{Gr}$ and $\mathrm{Pr}$
Ratio of the momentum and thermal diffusivities

Ratio of inertial forces to viscous forces

Ratio of buoyancy forces to viscous forces

\section{Remarks}

For small $\operatorname{Re}\left(\operatorname{Re} \leqslant 5 \times 10^{5}\right)$ the flow is laminar. With increasing $R e$ the transition to turbulence takes place accompanied by an increase in convective heat transfer

Typical values of $G r$ for microstructures are on the order of $10^{-8}$ to $10^{-2}$. Higher $\mathrm{Gr}$ indicates an increase in convective heat transfer

For small $P r$, heat diffuses faster than momentum, i.e. thermal boundary layer is shorter than velocity boundary layer

If $R a$ is smaller than a certain critical value for that fluid, the dominant heat transfer mechanism is conduction and if that critical value is exceeded, then convection takes over. Typical values of $R a$ for microstructures are on the order of $10^{-9}$ to $10^{-3}$

Nusselt number $\quad N u=\frac{h L}{k}=C R a^{n / m} \quad \begin{aligned} & \text { Dimensionless temperature } \\ & \text { gradient at the surface }\end{aligned}$

It is treated as a measure of the convective heat transfer occurring at the surface

$V\left(\mathrm{~m} \mathrm{~s}^{-1}\right)$ is the fluid velocity; $L(\mathrm{~m})$ is the characteristic length and $v\left(\mathrm{~m}^{2} \mathrm{~s}^{-1}\right)$ is the kinematic viscosity of the fluid; $g\left(\mathrm{~m} \mathrm{~s}^{-2}\right)$ is the gravitational acceleration; $\beta\left(\mathrm{K}^{-1}\right)$ is the volumetric thermal expansion coefficient; $\alpha\left(\mathrm{m}^{2} \mathrm{~s}^{-1}\right)$ is the thermal diffusivity; $h\left(\mathrm{~W} \mathrm{~m}^{-2} \mathrm{~K}^{-1}\right)$ is the convection coefficient; $k\left(\mathrm{~W} \mathrm{~m}^{-2}\right)$ is the thermal conductivity; $C, n$ and $m$ are constants with values depending on the range of $R a$ and other factors.

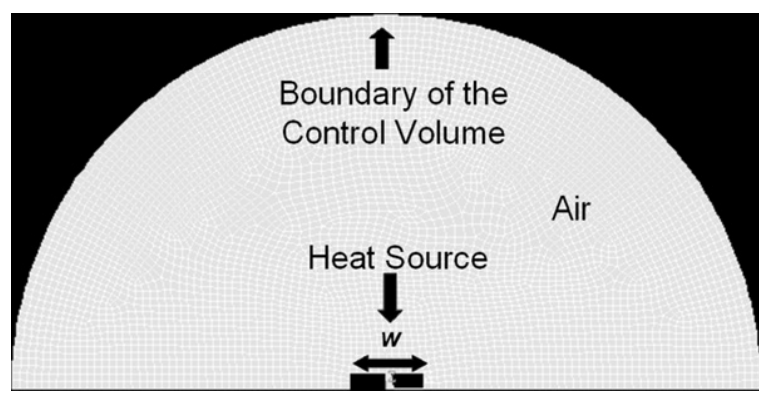

Figure 1. Control volume employed in the comparison of micro and macroscale heat transfer.

performed with finite element software ANSYS. Since $G r$ and $R a$ are directly proportional to gravity, gravity is introduced as a control parameter for the effect of buoyancy forces and hence, free convection. The problem geometry is given in figure 1 and consists of a heat source surrounded by a fluid or solid medium with varying dimensions, where the scale effect is introduced. Temperatures of the heat source and outer boundary of the surrounding medium are set constant. The resulting velocity and heat flux distributions inside the surrounding medium are reported at different time points allowing the observation of their evolution. Transient thermal analyses in a fluid medium are performed for both micro- and macroscales. Then the same geometry and boundary conditions are applied to the solid model at microscale, where only conductive heat transfer mechanism is considered. Details of the fluid and solid models in a finite control volume are given in appendix A. Appendix B describes the solid model where the dimensions of the surrounding medium are extended to infinity. This aspect will later be useful in determining heat transfer coefficients in the case of a microdevice surrounded by air.
Table 2 provides a list of cases considered and a partial summary of simulation results in terms of maximum fluid velocity and heat flux. For microscale analysis, one can refer to figure 2 for a full description of obtained results. It shows the time-dependent heat flux, $q^{\prime \prime}$, in solid and fluid media (both taken to be air at atmospheric pressure) with and without a gravitational field. The first and most noticeable observation is that heat flux does not change when gravity condition is imposed in the fluid environment. Contour plots for heat flux around the source at $t=10 \mu \mathrm{s}$ and $t=1 \mathrm{~ms}$ are independent of the gravitational condition. Although contour plots for velocity computed for both cases at $t=10 \mu \mathrm{s}$ and $t=1 \mathrm{~ms}$ seem to be different, this difference remains on the order of only $10^{-6} \mathrm{~m} \mathrm{~s}^{-1}$ (table 2). The explanation lies in the dimensions of the system. Due to the small size of the system, density variations are very small in the control volume, and thus gravitational forces do not become significant. This shows that at microscale, free convection is not dominant and conduction should be treated as the only mode of heat transfer at moderate temperatures.

Another important observation from figure 2 is about the same temporal evolution of heat flux through both conductive modeling of air with solid elements and convective modeling with fluid elements. However, heat flux values are higher for solid modeling than those obtained through fluid modeling. Furthermore, an increase in this difference with increasing temperature is also evident in figure 2, when simulation results for $T=500 \mathrm{~K}$ and $T=800 \mathrm{~K}$ are compared. This is a clear indication that modeling with free convection underestimates the actual heat transfer at microscale as stated previously by Guo et al [9]. This observation confirms further that modeling heat transfer from the exposed surfaces of a microdevice with only conduction is more appropriate than a free-convectionbased approach. 

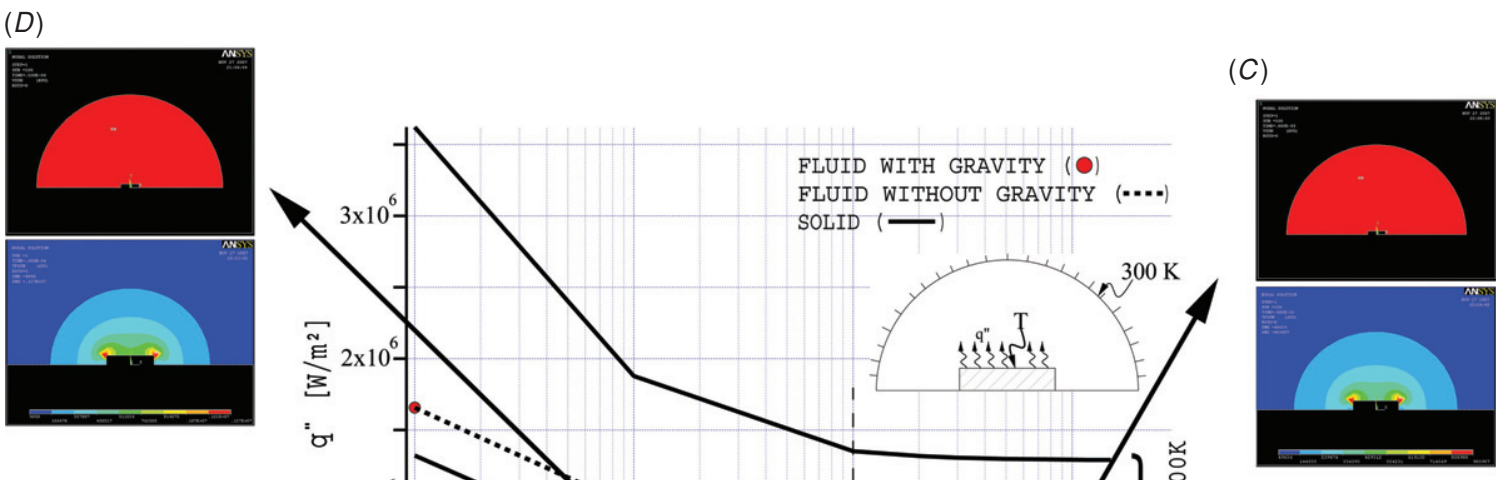

(A)

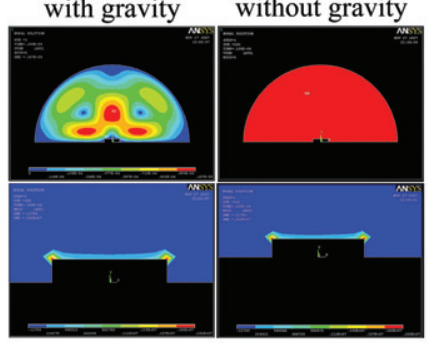

Figure 2. Plot of heat flux, $q^{\prime \prime}$, versus time, $t$, at microscale for solid and fluid media at source temperatures of $500 \mathrm{~K}$ and $800 \mathrm{~K}$. The snapshots of velocity (top) and heat flux (bottom) distributions are shown: $(A)$ fluid medium with and without gravity after $10 \mu \mathrm{s} ;(D)$ solid medium after $10 \mu \mathrm{s} ;(B)$ fluid medium with and without gravity after $1000 \mu \mathrm{s} ;(C)$ solid medium after $1000 \mu \mathrm{s}$.

Table 2. A list of cases considered in the transient thermal analyses along with characteristic numbers for maximum velocity and heat flux. Time refers to the duration of heating. Node 205 corresponds to the midpoint of the source/air interface, whose length is designated by $w$ in figure 1.

\begin{tabular}{|c|c|c|c|c|c|c|}
\hline \multirow[b]{2}{*}{ Microscale } & \multicolumn{2}{|c|}{ Element type } & \multirow{2}{*}{$\begin{array}{l}\text { Time } \\
10 \mu \mathrm{s} \\
1 \mathrm{~ms}\end{array}$} & \multirow{2}{*}{$\begin{array}{l}\begin{array}{l}\text { Maximum fluid } \\
\text { velocity }\left(\mathrm{m} \mathrm{s}^{-1}\right)\end{array} \\
0.107 \times 10^{-5} \\
0.131 \times 10^{-5}\end{array}$} & \multirow{2}{*}{$\begin{array}{l}\text { Heat flux at } \\
\text { node } 205\left(\mathrm{~W} \mathrm{~m}^{-2}\right) \\
393850 \\
314850\end{array}$} & \multirow{2}{*}{$\begin{array}{l}\text { Conclusion for heat flux } \\
\text { (1) Decreasing heat flux with } \\
\text { increasing time }\end{array}$} \\
\hline & Fluid (Fluid141) & Gravity & & & & \\
\hline & & No gravity & $\begin{array}{l}10 \mu \mathrm{s} \\
1 \mathrm{~ms}\end{array}$ & $\begin{array}{l}0 \\
0\end{array}$ & $\begin{array}{l}393890 \\
314900\end{array}$ & (2) No effect of gravity \\
\hline & $\begin{array}{l}\text { Solid (Plane 55 + } \\
\text { Infin110) }\end{array}$ & Finite boundary & $\begin{array}{l}10 \mu \mathrm{s} \\
1 \mathrm{~ms}\end{array}$ & Not relevant & $\begin{array}{l}652410 \\
409480\end{array}$ & $\begin{array}{l}\text { (1) Decreasing heat flux with } \\
\text { increasing time (similar to a } \\
\text { fluid) }\end{array}$ \\
\hline & & Infinite boundary & $\begin{array}{l}10 \mu \mathrm{s} \\
1 \mathrm{~ms}\end{array}$ & & $\begin{array}{l}653060 \\
30797\end{array}$ & \\
\hline \multirow[t]{2}{*}{ Macroscale } & Fluid (Fluid141) & Gravity & $\begin{array}{l}1 \mathrm{~ms} \\
1 \mathrm{~s}\end{array}$ & $\begin{array}{l}0.239 \times 10^{-3} \\
0.238\end{array}$ & $\begin{array}{l}5.8082 \\
447.90\end{array}$ & $\begin{array}{l}\text { (1) Increasing heat flux with } \\
\text { increasing time in the presence } \\
\text { of gravity }\end{array}$ \\
\hline & & No gravity & $\begin{array}{l}1 \mathrm{~ms} \\
1 \mathrm{~s}\end{array}$ & $\begin{array}{l}0 \\
0\end{array}$ & $\begin{array}{l}5.5838 \\
5.5839\end{array}$ & $\begin{array}{l}\text { (2) Gravitational effect through } \\
\text { buoyancy forces }\end{array}$ \\
\hline
\end{tabular}

Macroscale analyses are similarly summarized in figure 3, which shows the time-dependent heat flux within a fluid medium. Once again the medium is chosen to be air at atmospheric pressure. An inset with the same time scale as the one of figure 2 is provided for comparison purposes. First of all, in contrast to the previous case, it is evident that heat flux changes considerably when the gravity condition is imposed in the fluid environment. Table 2 shows two orders 

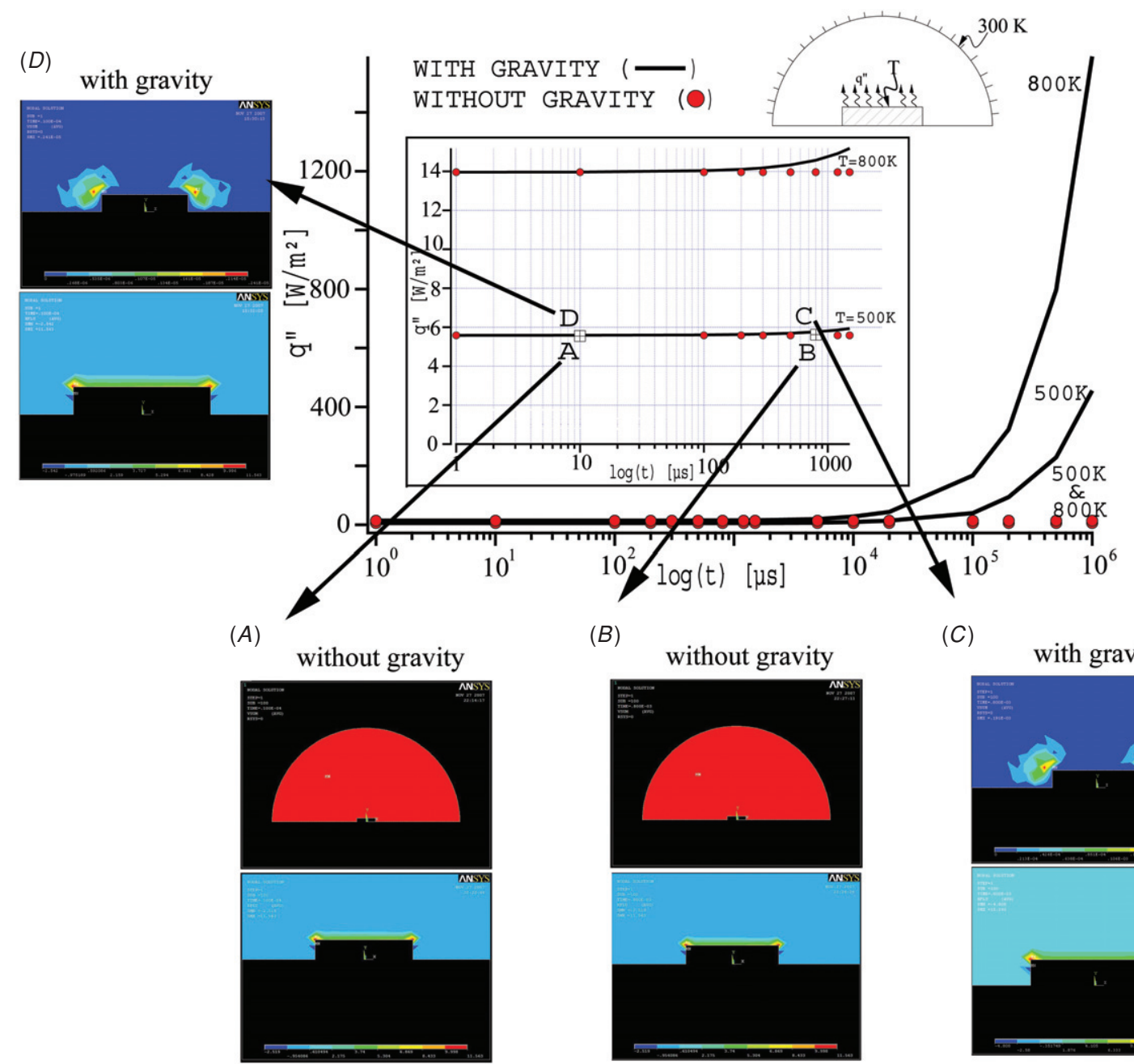

$(B)$

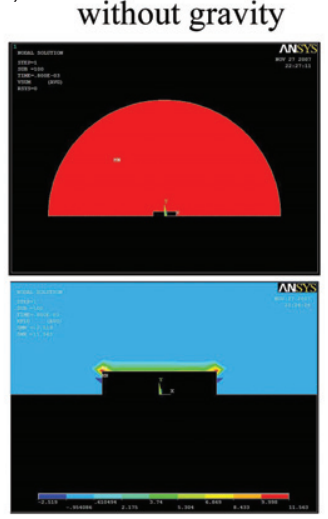

$(C)$

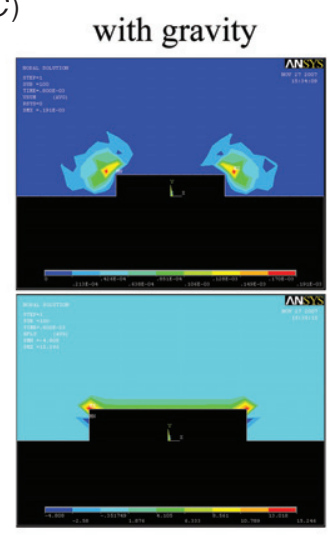

Figure 3. Plot of heat flux, $q^{\prime \prime}$, versus time, $t$, at macroscale for a fluid medium. The snapshots of velocity (top) and heat flux (bottom) distributions are shown: $(A)$ fluid medium without gravity after $10 \mu \mathrm{s} ;(B)$ fluid medium without gravity after $1000 \mu \mathrm{s} ;(C)$ fluid medium with gravity after $1000 \mu \mathrm{s} ;(D)$ fluid medium with gravity after $10 \mu \mathrm{s}$.

of magnitude difference in heat flux for a $1 \mathrm{~s}$ duration of heating. This is mainly due to the fact that density variations, thus the density gradient, in the control volume are high and the gravitational effects, thus the body forces acting on air elements, are considerable. This, in turn, increases the bulk velocity of air causing convective effects to be dominant at macroscale. However, if we exclude gravity from the analyses, the density gradient does not result in a gravitational force, prevents air from gaining velocity and as a result, velocity does not develop on top of the surface and the resulting heat flux remains low. This shows that at macroscale, free convection is the dominant type of heat transfer mode.

Another conclusion arising from a comparison of figures 2 and 3 is the efficiency of thermal actuation at microscale when compared to macroscale. At microscale, although heat flux is very high at the beginning, it decreases rapidly with time and attains a steady-state value which is a minimum in the plot given in figure 2 . In contrast, heat flux at macroscale shows a divergent behavior as evident in figure 3. This has direct implications in energy consumption and shows why thermal actuation is a preferred method at microscale.

\subsection{Review of modeling approaches}

Having determined the importance of length scale in heat transfer, it would be beneficial to conduct a critical review of the literature on the modeling of microscale thermal actuators. Special emphasis will be placed on the use of free convection and conduction while studying heat transfer through microdevice/air interfaces.

In deciding the operating frequency of a photothermal microactuator, Baglio et al [10] took the dominant factor in system dynamics to be the cooling time of the actuator, and this cooling was assumed to occur due to free convection on the device surface. To decide the average convection coefficient, the procedure that uses equation (1) was employed. However, it is important to note that the correlation used to decide $\mathrm{Nu}$ from $R a$ should specifically be applicable in the $R a$ range of the microactuator in question. Guidelines for the correct approach can be found in [6].

Mankame and Ananthasuresh [11] also used empirical correlations between $\mathrm{Nu}$ and $\mathrm{Ra}$ given by Mills [12] to calculate the temperature and size-dependent convection coefficients. Since the correlations are not defined for the $R a$ range of 
flat surfaces of the microstructures, these correlations were extrapolated in [11]. Due to the fact that the criterion for free convection lies with $R a$ and $R a$ is considerably small for buoyancy forces to overcome viscosity, the applicability of the mentioned model is limited. The same approach, i.e. applying purely convective heat transfer on all surfaces of the microactuator, was employed in a variety of other thermal modeling studies (Jungen et al [13], Chen et al [14], Todd and Xie [15], Yang and Yu [16]).

Similarly, the working principle of a convective accelerometer was based on the sensing of a temperature difference that is caused by a change in free convective flow due to acceleration (Milanovic et al [17] and Luo et al [18]). Free convective flows were caused by the buoyant forces generated by the thermal difference between a heated element in the device and the surrounding gas. Although acceleration might cause convective flows, this would be forced convection rather than free convection. (Similar to previous cases, it should be remembered that in small scale, gravitational effects, thus, the buoyant forces, would be negligible.) Therefore the relation between the measured temperature difference and the amount of acceleration is not to be explained by free convective effects. Hence, it is not surprising that a deviation from linearity for $G r$ below $10^{-4}$ was reported in [18], which is a typical value for microscale devices.

In 2003, Guo et al [9] mentioned that for $R a<10^{3}$ free convective effects should begin to cease and conduction should become the only heat transfer mechanism due to negligible inertial forces at small scale. The study also indicated that if the conventional correlations are used for free convection at microscale, the actual amount of heat transferred might be underestimated as indicated in figure 2.

Similarly, in 2002 Hickey et al [19] emphasized the relation between $R a$ and free convection, and concluded that free convection was important at macroscale. In this study, a Guckel-type actuator was modeled with a small gap between the actuator and the substrate surface. The conduction heat transfer was taken to be limited to this gap only, while thermal boundary conditions on all other surfaces were omitted. By doing so, the model considered the scale of the air gap rather than the size of the device. This technique has been widely accepted by other studies as well (Maloney et al [20], Lott et al [21], Huang and Lee [22], Serrano et al [23], Li and Uttamchandani [24] and Yan et al [25]).

In a similar problem, Geisberger et al [26] stated that low $G r$ was an indication of the dominance of conduction on the surfaces of microscale structures. A method for 3D finite element modeling (FEM) of thermal microstructures, particularly a Guckel actuator, was proposed. The thin film of air both between the arms of the actuator and between the bottom of the actuator and the substrate was modeled with conductive air elements. Convection coefficients were assigned to the surfaces that were exposed to the open atmosphere. The coefficients were determined via Flotran CFD analysis in ANSYS for an infinite plate. Other works such as Kuang et al [27], Zhang et al [28], Li et al [29], Huang et al [30] and Atre [31] also used a combination of conductive and convective heat transfer modes in their analyses.
Finally, in a recent study by Solano et al [32] similar conclusions were drawn by comparing $R a$ at microscale with that at macroscale, and conduction was employed as the only mode of heat transfer mechanism to the ambient environment at microscale.

From this review on a rather narrow spectrum of devices, it becomes clear that no consensus is established in the field of the modeling of thermal microsystems. In the remainder of this work, a methodology will be introduced for the assignment of temperature- and scale-dependent conductive heat flux on the surfaces of thermal actuators. The methodology will then be applied to a bimorph actuator.

\section{Methodology}

Having confirmed the importance of conduction in microscale heat transfer, the appropriate conductive heat transfer coefficients to be applied on the surfaces of thermal microdevices should be extracted from the solid modeling of air.

As described by Kakac et al [8], for considerably thick solids with a heat source on their surface, the temperature of the solid remains at the ambient temperature after a finite thickness called the penetration depth at a certain time, $\delta(t)$. Similarly, due to the fact that the ambient environment acts as a thermal reservoir, it is safe to assume that at thermal equilibrium there is a finite penetration depth, $\delta$, for a microdevice with a temperature profile higher than the ambient temperature. Finding $\delta$ requires knowledge of the thermal diffusivity of the material, of which the surrounding medium is made of, and the thermal time constant, $\tau_{\text {thermal }}$, of the microdevice, i.e. the time scale at which the temperature of the device attains equilibrium with the atmosphere. An indication of $\tau_{\text {thermal }}$ is provided in figure 2 . On the other hand, experiments based on the measurement of actuator displacements can only yield the mechanical time constant, $\tau_{\text {mech. }}$ Since it would be impossible for the device to attain mechanical equilibrium before it reaches a steady-state temperature, $\tau_{\text {mech }}$ can safely be considered as an upper bound for $\tau_{\text {thermal }}$.

A time-dependent semi-infinite model of the conductive heat transfer in solids provides the necessary context for the computation of $\delta$. Simulation details of the semi-infinite model can be found in appendix B. To demonstrate the concept, figure 4 shows the temperature distribution in semi-infinite air and $\mathrm{Al}$ media kept in contact with a $500 \mathrm{~K}$ temperature source for $2.5 \mu \mathrm{s}$. Since the thermal diffusivity of air is less than that of $\mathrm{Al}$, ambient temperature, $300 \mathrm{~K}$, is reached at a shorter penetration depth indicating that $\delta_{\text {air }}$ is shorter than $\delta_{\mathrm{Al}} \cdot \tau_{\text {thermal }}$ can be calculated for this penetration depth using equation (2) [8], where $\delta(\mathrm{m})$ is the penetration depth and $\alpha\left(\mathrm{m}^{2} \mathrm{~s}^{-1}\right)$ is the thermal diffusivity. In this model, as time progresses, the overall temperature should uniformly reach the source temperature at the steady state. However, in the case of a microactuator embedded in an infinite medium, although a conductive model for air is utilized, the solid medium resembles a good insulator with a point source. In this case, it is safe to assume that the ambient temperature is reached very rapidly as one moves away from the source. This indicates 


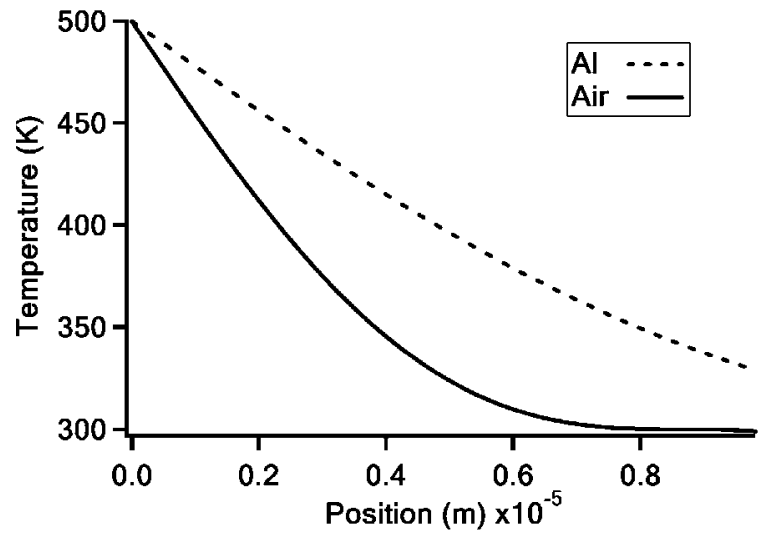

Figure 4. Temperature distributions in infinitely extending air and aluminum media in contact with a $500 \mathrm{~K}$ temperature source for $2.5 \mu \mathrm{s}$. Horizontal axis represents the distance from the temperature source.

that $\tau_{\text {thermal }}$ is very low and temperature gradients are confined to a narrow strip around the source, known as the boundary layer:

$$
\tau_{\text {thermal }}=\frac{\delta^{2}}{\alpha} .
$$

If $\tau_{\text {thermal }}$ was known, a transient analysis could be performed to find $\delta$ approximately. $\delta\left(\tau_{\text {thermal }}\right)$ could then be accepted as the penetration depth and conduction coefficients, and hence the heat flux to be used in electro-thermal modeling of the device could be found. However, measurement of $\tau_{\text {thermal }}$ on the order of nanoseconds is a difficult task. Since displacement of microactuator is usually measured rather than the temperature distribution over the device, an alternative path is taken in this study. Based on the previous argument, $\tau_{\text {mech }}$ is taken as an upper limit for $\tau_{\text {thermal }}$ leading to the conclusion that the heat flux in the system will not change after $\tau_{\text {mech }}$. Then, a conductive heat transfer simulation is performed within the context of figure 2 to find heat flux for different sizes of boundary layers. These heat flux values are then applied as boundary conditions in device simulations. Finally, obtained results can be compared to experimental measurements, where the best fit indicates the correct penetration depth.

The major focus of this approach is placed on the application of conductive heat flux as a thermal boundary condition in the finite element analysis. To accomplish this, convective thin film coefficients, an inherent property of various commercial finite element software packages, are utilized instead of introducing a new, external function. This ensures a wide applicability of the proposed method in a variety of computational platforms. The approach is based on imposing heat flux, $q^{\prime \prime}=k(\partial T / \partial x)$, as a boundary condition, and using the convective thin film coefficient, $h$, as an intermediate tool to assign $q^{\prime \prime}$. Equating $q^{\prime \prime}$ in conductive and convective modes, one obtains $h=\frac{k}{T_{s}-T_{\infty}} \frac{\partial T}{\partial x}$, where $T_{s}$ is the surface temperature and $T_{\infty}$ is the ambient temperature. Hence, the computational procedure for establishing a list of $h$ is as follows:

(1) computation of the heat flux at different temperatures in a solid modeling similar to the one described in the context of figure 2 ,

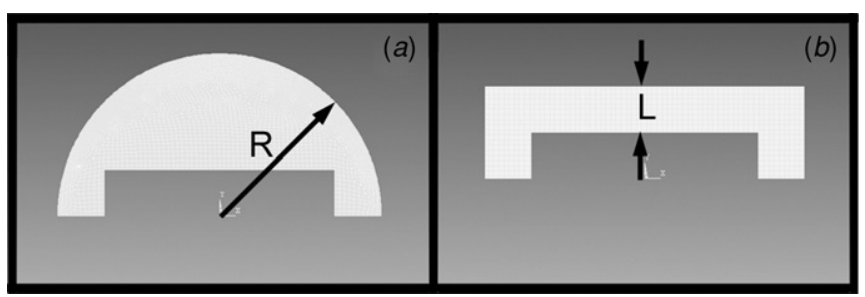

Figure 5. (a) Spherical boundary layer; $(b)$ rectangular boundary layer.

(2) computation of the corresponding thin film coefficients, and

(3) construction of the function of $h$ for various temperatures.

The geometry of the boundary layer used for the computation of temperature-dependent conduction coefficients is shown in figure 5. The critical dimension ( $w$ in figure 1) is taken to be $10 \mu \mathrm{m}$. For boundary layer thicknesses larger than $2 \mu \mathrm{m}$, radial geometries are used (figure 5(a)) and for $2 \mu \mathrm{m}, 1 \mu \mathrm{m}$ and $0.5 \mu \mathrm{m}$ thick boundary layers rectangular geometries are used (figure 5(b)). This distinction is due to meshing issues only and does not interfere with the actual heat transfer mechanism. The outer periphery not in contact with the heat source is taken to be at $300 \mathrm{~K}$. This procedure can be repeated for other device sizes and thus, the corresponding set of coefficients can be obtained.

Dimensions of the boundary layers and the resulting temperature-dependent heat transfer coefficients, $h$, are listed in table 3. Numbers listed in table 3 can be used for the general purpose of modeling heat transfer from a microdevice (with a critical dimension of $10 \mu \mathrm{m}$ ) to the surrounding air at atmospheric pressure. To achieve this, a prior knowledge of the boundary layer thickness is necessary, so that one knows which column in table 3 to utilize. An experimental procedure addressing this issue will be provided in the next section.

It is also evident in table 3 that, for a given control volume, $h$ increases convergently with increasing temperature agreeing well with the concept of power efficiency of thermal actuation at microscale. This is fundamentally different and in contrast to the convection coefficients proposed by Mankame and Ananthasuresh [11] that exhibit a divergent behavior as a function of temperature.

As a result, the proposed methodology consists of the following steps:

(1) determination of the thermal time constant of the microactuator of interest;

(2) determination of the boundary layer size associated with this thermal time constant using equation (2);

(3) extraction of appropriate thin film coefficients from table 3 (construction of the $h$ function should be repeated for critical dimensions other than $10 \mu \mathrm{m}$ );

(4) application of the listed values in table 3 as boundary conditions in thermomechanical finite element simulations. 
Table 3. Temperature-dependent heat transfer coefficients $(h)$ for different penetration depths, $\delta$. The critical dimension of the device is taken to be $10 \mu \mathrm{m}$.

\begin{tabular}{lllllr}
\hline & \multicolumn{5}{l}{ Temperature-dependent $h\left(\mathrm{~W} \mathrm{~K}^{-1} \mathrm{~m}^{-2}\right)$ values for different boundary layers } \\
\cline { 2 - 6 } Temperature (K) & $\begin{array}{l}R^{\mathrm{a}}=100 \mu \mathrm{m} \\
h\end{array}$ & $\begin{array}{l}R=14 \mu \mathrm{m} \\
h\end{array}$ & $\begin{array}{l}L^{\mathrm{b}}=2 \mu \mathrm{m} \\
h\end{array}$ & $\begin{array}{l}L=1 \mu \mathrm{m} \\
h\end{array}$ & $\begin{array}{l}L=0.5 \mu \mathrm{m} \\
h\end{array}$ \\
\hline 300 & 1808.6 & 11217 & 15186 & 28357 & 54841 \\
500 & 2198.8 & 13821 & 18757 & 34981 & 67608 \\
700 & 2552.7 & 16271 & 22030 & 41192 & 79617 \\
900 & 2895.9 & 18663 & 25265 & 47253 & 91320 \\
1100 & 3139.5 & 20450 & 27711 & 51746 & 100120 \\
1300 & 3285.7 & 21529 & 29169 & 54477 & 105360 \\
1500 & 3384 & 22249 & 30140 & 56311 & 108960 \\
1700 & 3454.9 & 22764 & 30833 & 57615 & 111380 \\
1900 & 3508.7 & 23152 & 31352 & 58585 & 113440 \\
2100 & 3550.8 & 23452 & 31758 & 59345 & 114720 \\
\hline
\end{tabular}

${ }^{a} R$ stands for a spherical boundary layer (figure $5(a)$ ).

${ }^{\mathrm{b}} L$ stands for a rectangular boundary layer (figure $5(b)$ ).

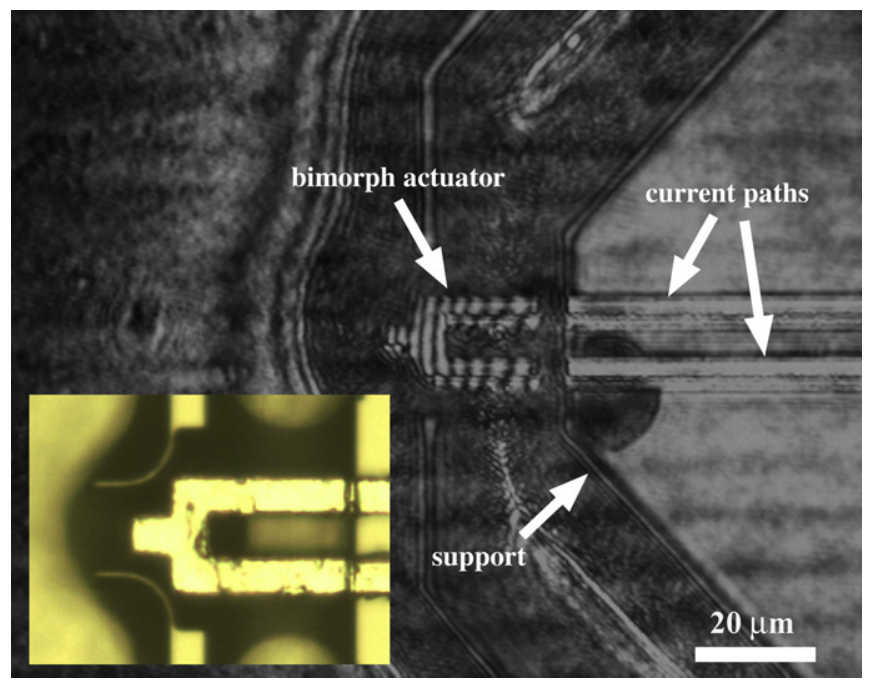

Figure 6. A bent bimorph actuator viewed under a home-made microscope. Fringe pattern on the surface is an indication of displacement. The inset shows the same actuator before excitation.

\section{A case study: bimorph actuation}

In this section the approach described above will be applied to a bimorph thermal actuator on a Si substrate shown in figure 6. The objective is to establish a procedure to determine the thermal time constant. The design has a critical length of $10 \mu \mathrm{m}$. The upper layer of the actuator is designed to be a $1 \mu \mathrm{m}$ thick Al film. The bottom layer is polyimide (PI) of $2 \mu \mathrm{m}$ thickness. The device is separated from the $\mathrm{Si}$ substrate by a $2.5 \mu \mathrm{m}$ thick $\mathrm{SiO}_{2}$ layer. Following the determination of conductive heat flux as described in the previous section, voltage versus displacement simulations are performed by using the $h$ function as the thermal boundary condition on the surfaces of the thermal microactuator. Details of displacement simulations are given in appendix C. In the following, the device fabrication will be explained first. Static and dynamic measurements of device displacement will be discussed and the section will be concluded with a comparison of experimental and simulated displacement results leading
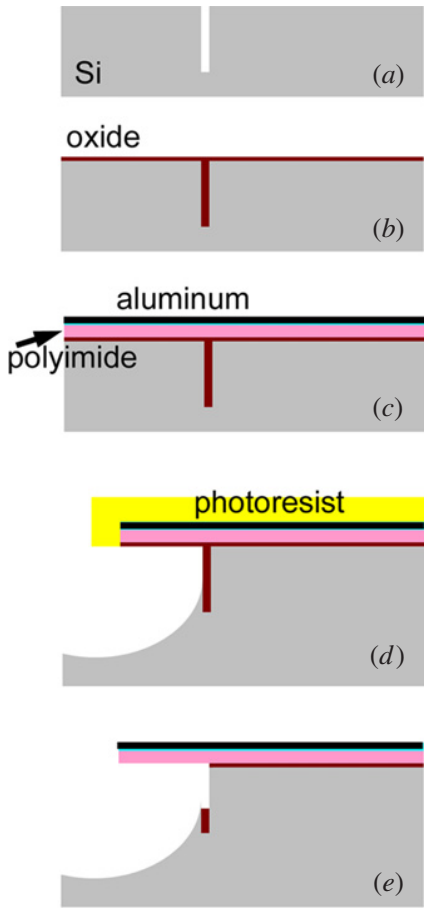

Figure 7. Fabrication sequence.

to the determination of the corresponding boundary layer thickness and thermal time constant.

\subsection{Device fabrication}

Fabrication of the thermal microactuator is a three-mask process utilizing conventional UV photolithography with positive photoresist. The first and third masks are used for the release process and the second mask is used to define the actual shape of the thermal microactuator.

The process starts with a bulk silicon wafer. The trench defining the release area underneath the cantilever is opened with the deep reactive ion etching (DRIE) process. After DRIE and resist removal (figure 7(a)), the top surface of the wafer and the side walls of the trenches are covered with 


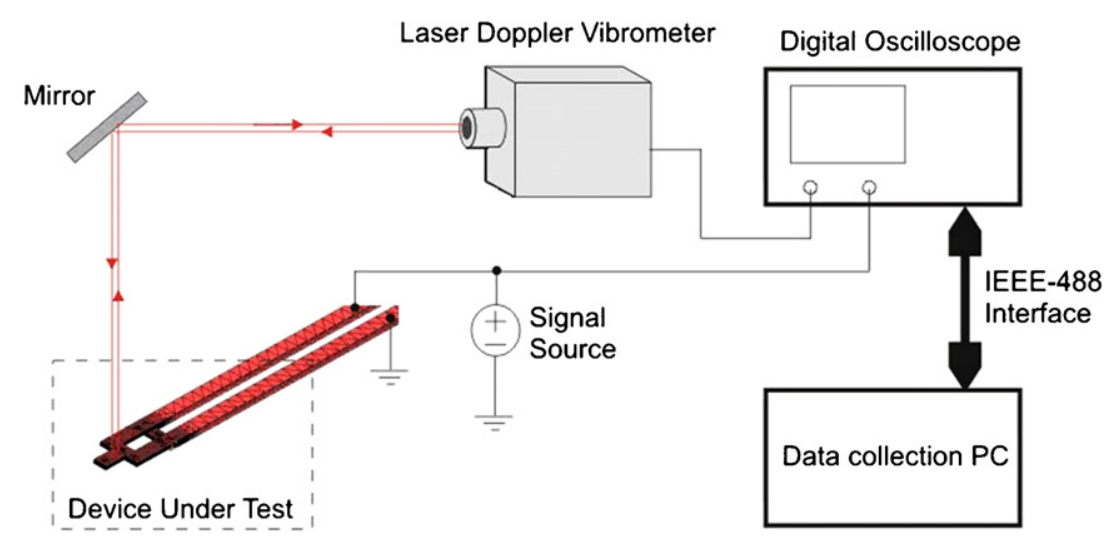

Figure 8. Setup used for the determination of the mechanical time constant, $\tau_{\text {mech }}$.

thermal oxide and low temperature oxide (LTO) which is reflown at $1050{ }^{\circ} \mathrm{C}$ for $15 \mathrm{~min}$ (figure $7(b)$ ). Here, thermal oxide serves as an etching barrier during the isotropic Si etch in the release process and LTO fully fills the gap between trenches yielding a smooth surface during polyimide PI2610 (HD Microsystems ${ }^{\mathrm{TM}}$ ) spin coating. After oxidation, PI-2610, which is the bottom layer of the thermal actuator, is spin-coated and cured. Then its thickness is reduced to $2 \mu \mathrm{m}$ with $\mathrm{O}_{2}$ plasma etching. Next, a $1 \mu \mathrm{m}$ thick $\mathrm{Al}$ film is sputtered as the top layer of the thermal actuator (figure $7(c)$ ). Second UV exposure is carried out to define the thermal actuator. Aluminum, PI and oxide layers are directionally etched with $\mathrm{Cl}_{2} / \mathrm{BCl}_{3}, \mathrm{O}_{2}$ and $\mathrm{CF}_{4}$ plasmas, respectively. Finally, photoresist is stripped off.

The final step of the fabrication is the release of the thermal actuator. Using a third lithography mask, the body and the tip of the thermal actuator are covered with a positive photoresist. Then anisotropic Si etching with ICP-DRIE is performed. This is followed by isotropic Si etching with $\mathrm{SF}_{6}$ plasma to release the mobile tip of the thermal microactuator (figure $7(d)$ ). As previously mentioned, thermal oxide serves as an etch stop for the isotropic Si etch. Next, an isotropic oxide etch is carried out to etch the oxide layer below the released part of the thermal actuator. After this step, the bimorph thermal microactuator is completely defined and released from $\mathrm{Si}$. The final step is the stripping of the photoresist (figure 7(e)).

\subsection{Device characterization}

An interferometric lens mounted on a Nikon Eclipse LV100 microscope is used for static deflection characterization of the thermal actuator in ambient air. The device is probed by using manipulators under the $10 \times$ interferometric objective (Mirau type, numerical aperture: 0.13). A dc voltage is applied and the number of fringes due to the deflection of the actuator tip are counted. Red light with a wavelength of $\lambda=655 \mathrm{~nm}$ is used for illumination. Each fringe pattern of the acquired image corresponds to the half wavelength and therefore the resolution for the out-of-plane displacement measurement is approximately $163 \mathrm{~nm}(\lambda / 4)$. An example can be seen in figure 6.

Dynamic testing of the thermal actuator is performed in ambient air using a custom-built setup sketched in figure 8 .

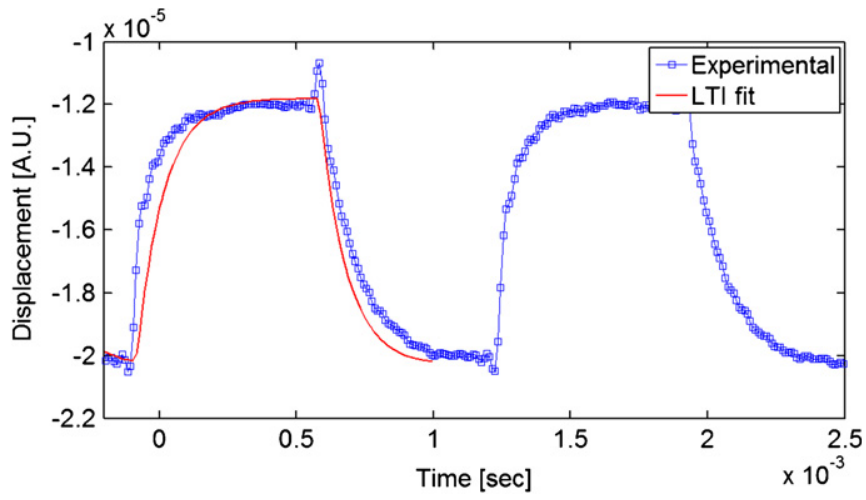

Figure 9. Measured transient behavior of the actuator.

The setup is composed of a Laser Doppler Vibrometer (LDVPolytec PDV100) which detects the velocity of vibrating structures, a signal source for actuation, an oscilloscope which allows real-time measurement of the velocity as well as a data collection PC where the input signal and the displacement signal are stored. Actuation of the device exerts an outof-plane displacement of the actuator tip; thus a mirror is incorporated in the setup to both direct the laser beam of the LDV to the device and to collect the reflected light into the LDV. In order to measure the deflection dynamics of the device, a pulse signal is applied to the electrodes and the resulting velocity profile is collected at the oscilloscope. Deflection of the actuator is obtained by time integration of the velocity profile. Figure 9 shows the deflection of the actuator along with a fit function modeling the second-order, linear time invariant (LTI) system response due to the forceto-displacement transfer function of the mechanical structure. Step response of the actuator shows a critically damped system due to air damping resulting in a rise time of $240 \mu$ s and a fall time of $600 \mu \mathrm{s}$. This rise time is used as the mechanical time constant, $\tau_{\text {mech }}$, throughout the simulations.

\subsection{Comparison of simulation and experiment for static measurements}

Figure 10 gives the comparison of experimental and simulated results for the static test. Normalized displacement $\left(d / l^{2}\right)$ 


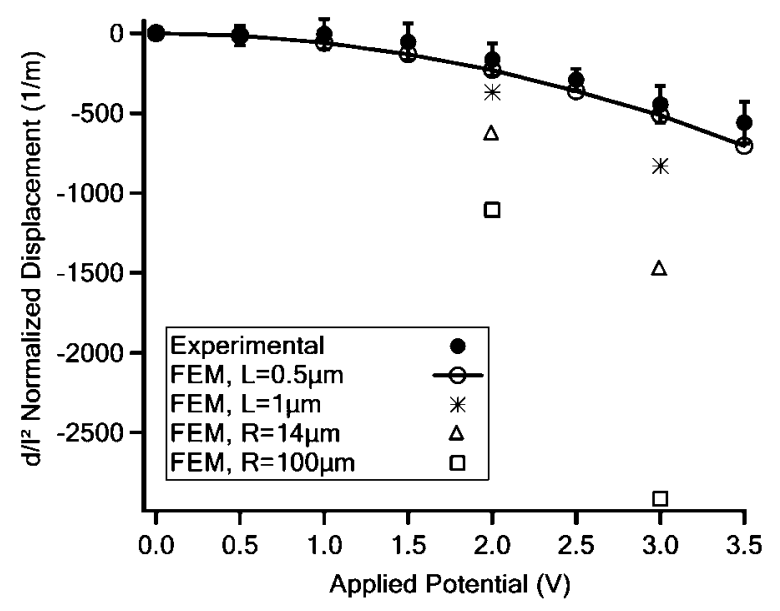

Figure 10. Experimental versus simulated normalized displacement results as a function of applied potential.

is plotted as a function of the applied dc voltage. Since experiments are performed on three samples with different lengths, $l$, comparison is made over the mean of the normalized displacements, where $d$ is the tip displacement.

According to figure 10, as the boundary layer thickness (see figure 5 for the geometry of radial $(R)$ and rectangular $(L)$ boundary layers) is reduced, simulated results approach experimental results. The mechanical time constant, $\tau_{\text {mech }}$, was measured to be $240 \mu \mathrm{s}$ as explained in the previous section using dynamic measurements. From the semi-infinite approach, this corresponds to a penetration depth, $\delta$, less than $100 \mu \mathrm{m}$. That is why simulations are carried out starting with a $100 \mu \mathrm{m}$ thick boundary layer which is gradually decreased until experimental results for displacements are achieved. Experiment and simulation coincide only when $L$ is reduced from $100 \mu \mathrm{m}$ all the way to $500 \mathrm{~nm}$. $\tau_{\text {thermal }}$ can be calculated for this penetration depth using equation (2), where $\delta$ is equal to $L$ in this case. As a result, the thermal equilibrium is observed to be reached in around $10 \mathrm{ns,}$, which, as expected, is very rapid compared to $\tau_{\text {mech }}$. Hence, the last column in table 3 can be used to define boundary conditions in finite element simulations. This procedure has, of course, to be repeated for critical dimensions other than $10 \mu \mathrm{m}$.

Rapid heat transfer at small scale was mentioned by Feynman in his 1960 speech 'There's Plenty of Room at the Bottom' [33], where the need for cooling of moving parts in contact was questioned when the rate of heat transfer increases with decreasing scale. Due to the absence of convective flows at microscale, the only mechanism of heat loss to the ambient environment becomes conduction at moderate temperatures. Although the actuator attains thermal equilibrium with the surrounding atmosphere very quickly, it takes much longer to attain mechanical equilibrium due to the characteristics of the critically damped system.

\section{Conclusions}

In the first part of this manuscript, the scale dependence of heat transfer modes was studied. Finite element simulations of micro- and macroscale heat transfer phenomena were carried out in finite and infinite control volumes along with necessary comparisons with available analytical solutions. The following conclusions/verifications were achieved from this introductory section.

(1) The effect of gravity in heat transfer is negligible at microscale. This fact eliminates free convection as an alternative way of heat dissipation from a hot surface into air. Therefore, conduction should be treated as the only mode of heat transfer at microscale.

(2) A literature survey provided along with this study highlights the necessity of clarification regarding the dominance of conduction in the field of modeling of microscale thermal actuators.

The second part of the study was then geared toward establishing a methodology to incorporate heat conduction (instead of convection) in commercially available finite element software. To do this, a set of heat transfer coefficients was obtained as a function of temperature and boundary layer thickness. Instead of creating a new external function for the definition of boundary conditions via conductive heat transfer, already existing convective thin film coefficients were utilized. Keeping heat flux values constant, one was able to convert conduction boundary values into convective ones. A list of these coefficients was provided for different temperatures. The obtained list can be employed for any modeling work in air at atmospheric pressure for devices with a critical dimension of $10 \mu \mathrm{m}$.

Implications of this approach were demonstrated with a bimorph actuator. The following four-step procedure was proposed as a conclusion:

(1) determine the thermal time constant, $\tau_{\text {thermal, }}$ of the microactuator of interest;

(2) determine the boundary layer size associated with this thermal time constant;

(3) extract appropriate thin film coefficients from table 3;

(4) apply them as boundary conditions in thermomechanical finite element simulations.

Step 1, the determination of the thermal time constant, is the critical step in this procedure. It is a property difficult to measure. First, it can be very short. In fact, for a device with a critical dimension of $10 \mu \mathrm{m}$, a time constant of $10 \mathrm{~ns}$ was found. Second, for very small devices, temperature measurement tool might lack necessary resolution. Therefore the determination technique used in this work was based on fitting of experimental data obtained from static measurements. The same procedure can be applied to devices of different characteristic lengths, and a corresponding set of heat transfer coefficients can be obtained.

\section{Acknowledgments}

This work was supported in part by Koc University and by TUBITAK under grant no 104M216. C Ataman and Professor $\mathrm{H}$ Urey of Koc University are thanked for their help with characterization work. 


\section{Appendix A. Details of finite element analysis in scale comparison}

All fluid analyses in table 2 are carried out with twodimensional Fluid141 element available in Flotran CFD module in ANSYS. The medium for heat transfer is taken to be air. Velocity, pressure and temperature distributions are obtained from the conservation of momentum, mass and energy, respectively. As indicated in the ANSYS user manual [7], the matrix system derived from the finite element discretization of the governing equation for each degree of freedom is solved separately. The flow problem is nonlinear and the governing equations are coupled together. The sequential solution of all governing equations, combined with the update of any temperature or pressure-dependent properties, constitutes a global iteration. The target time is reached with $1 \mu$ s time steps with the number of global iterations per time step equal to 20 . Density, viscosity, conductivity and specific heat of air are introduced to the system. These properties are taken from [6]. Density variations are allowed. Before performing transient analyses, the system is initialized.

The control volume consists of a heat source in the middle surrounded by a circular region of air (figure 1). The interface between the heat source and air provides an interaction platform, whose size determines the scale of the problem. For simulations at microscale, this interaction surface is taken to be $10 \mu \mathrm{m}$ long (indicated as $w$ in figure 1), whereas for macroscale this length is increased to $10 \mathrm{~m}$. The temperature of air in contact with the interaction surface is assumed to be equal to the surface temperature, which is varied throughout the simulations. Similarly, a no-slip boundary condition is imposed and the velocity of air at that surface is set to zero. The temperature along the outer periphery of the control volume in figure 1 is set equal to $300 \mathrm{~K}$. The fluid velocities along these exterior nodes are set to zero as well. Since only free convection is considered, for which the viscous flow, i.e. the bulk motion of the fluid, is not important, the air velocity is assumed to be zero initially and stay as zero outside the control volume. Meanwhile, in the control volume, due to the conservation of momentum and energy, the air velocity may differ from zero after steady-state conditions are reached. However, with increasing distance from the interaction surface, the fluid velocity value should converge to zero in the case of free convection.

Gravitation is employed as a control parameter. The air medium through which the energy transfer takes place is modeled with and without gravity.

The motivation behind solid analysis is to directly compare fluid and solid modeling of air. For this purpose, the same geometry with similar temperature boundary conditions given in figure 1 is modeled except for the fact that this time gravitational effects and velocity distribution are of no concern. Modeling is carried out using a two-dimensional thermal solid Plane55 element. Once again, the medium for heat transfer is taken to be air. This particular element has the conduction capability with material properties such as density, conductivity and specific heat to be defined [7].

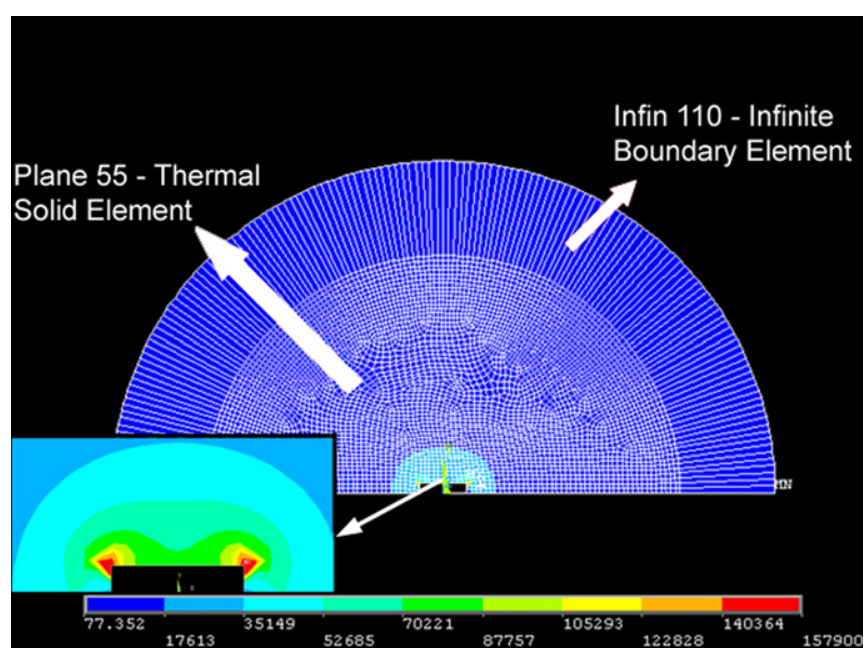

Figure B1. Finite element mesh for the study of conductive heat transfer for an infinitely extending medium. Infinite boundary element represents the open atmosphere. The inset shows the heat source and the details of the heat flux around it.

\section{Appendix B. Semi-infinite analysis}

An infinite solid Infin110 element is used to model an open boundary of a two-dimensional unbounded field problem [7]. A single layer of the element represents an exterior sub-domain of semi-infinite extent as shown in figure B1. The purpose of this study is twofold.

It serves the purpose of validating the employed numerical approach and mesh density by a comparison of simulation results with analytical solutions available for the semi-infinite model of conductive heat transfer for thick samples [8]. Since the comparison carried out with the specific mesh density of figure B1 is satisfactory, all analyses are performed with this mesh density.

In addition to validation, semi-infinite modeling also provides the context (equation (2)) for using temperaturedependent heat transfer coefficients with different boundary layer dimensions. These coefficients can then be utilized as boundary conditions while simulating the displacement of thermal microactuator as explained in section 4 and appendix $\mathrm{C}$.

\section{Appendix C. Details of finite element analysis in device simulations}

The $\mathrm{Si}$ substrate underneath the $\mathrm{SiO}_{2}$, on which the thermal actuator is placed, is not modeled in the finite element simulation. Since the thermal conductivity of air $\left(k_{\text {air }}\right)$ is very poor when compared to that of $\mathrm{Si}\left(k_{\mathrm{Si}} / k_{\mathrm{air}} \approx 6000\right), \mathrm{SiO}_{2}$ $\left(k_{\mathrm{SiO}_{2}} / k_{\text {air }} \approx 60\right)$ and PI $\left(k_{\mathrm{PI}} / k_{\text {air }} \approx 3\right)$, the major portion of the heat transfer takes place through the substrate rather than the surrounding air. Since the substrate is large compared to the actuator, it is considered as a thermal reservoir at ambient temperature and omitted from the simulations by applying a constant-temperature $(300 \mathrm{~K})$ boundary condition on the bottom surface of the $\mathrm{SiO}_{2}$ layer. Case studies, where the 


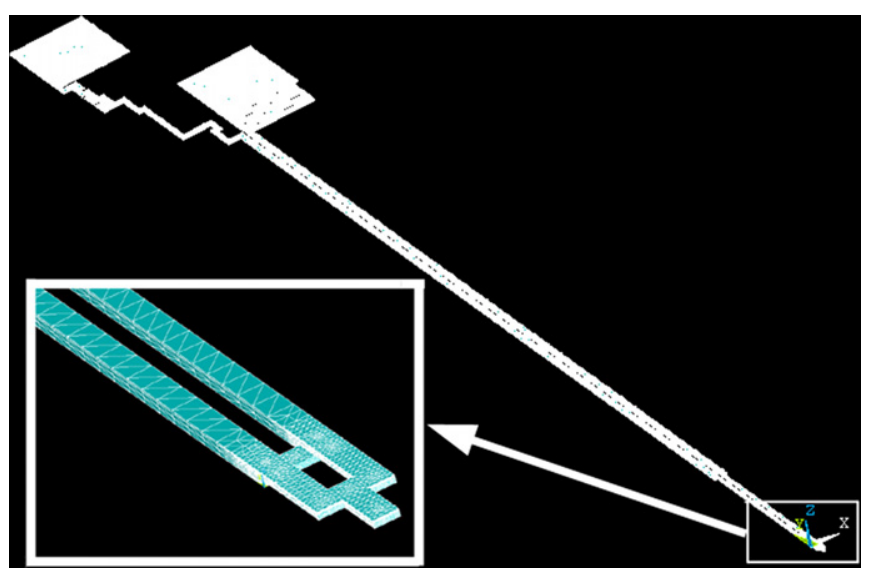

Figure C1. Overall device mesh. The inset depicts the refined mesh at the tip.

Table C1. Material properties.

\begin{tabular}{llll}
\hline & Aluminum & PI 2610 & $\mathrm{SiO}_{2}$ \\
\hline Elastic modulus $(\mathrm{GPa})$ & 70 & 8 & 73 \\
Poisson ratio & 0.35 & 0.2 & 0.17 \\
$\mathrm{CTE}(1 / \mathrm{K})(\mathrm{ppm})$ & 23.1 & 3 & 0.5 \\
Density $\left(\mathrm{kg} \mathrm{m}^{-3}\right)$ & 2700 & 500 & 2200 \\
Elect. resistivity $(\Omega \mathrm{m})$ & $26.5-94.7 \times 10^{-9}$ & $3 \times 10^{13}$ & $10^{13}$ \\
Thermal conductivity & 237 & 0.082 & 1.4 \\
$\left(\mathrm{~W} \mathrm{~m} \mathrm{~K}^{-1}\right)$ & & 1000 & 745 \\
Specific heat $\left(\mathrm{J} \mathrm{kg}^{-1} \mathrm{~K}^{-1}\right)$ & 900 & & \\
\hline
\end{tabular}

Table C2. Dimensions of a simulated bimorph actuator.

\begin{tabular}{lcll}
\hline & $\begin{array}{l}\text { Width } \\
(\mu \mathrm{m})\end{array}$ & $\begin{array}{l}\text { Thickness } \\
(\mu \mathrm{m})\end{array}$ & $\begin{array}{l}\text { Total length } \\
(\mu \mathrm{m})\end{array}$ \\
\hline Upper layer $(\mathrm{Al})$ & 10 & 1 & 9480 \\
Lower layer $(\mathrm{PI})$ & 10 & 2 & 9480 \\
Bottom layer $\left(\mathrm{SiO}_{2}\right)$ & 10 & 2.5 & 9386 \\
Anchor & 300 & - & 225 \\
\hline
\end{tabular}

substrate is included in the finite element model, confirmed its negligible effect on the simulation results. Hence, the substrate is excluded from the model for the rest of the study.

In addition, the actual geometry of the Al heater layer is preserved as shown in figure $\mathrm{C} 1$ because in a separate analysis the relation between the geometry of the heater layer and the current flux, and hence the temperature distribution on the actuator, is observed to be significant. The maximum element edge length is $10 \mu \mathrm{m}$. It is refined toward the tip, where the displacement is measured. Increasing the element edge by a factor of 2 changed neither the temperature nor the displacement profiles.

Material properties and geometries are given in tables $\mathrm{C} 1$ and $\mathrm{C} 2$, respectively. Electrical resistivity of aluminum, the heater layer, is taken to be temperature-dependent.

Device simulations are performed using the Solid 98 element. It is a ten-node, tetrahedral element with up to six degrees of freedom at each node and has a quadratic displacement behavior [7]. It is well suited to model irregular geometries. Voltage values ranging from $0.5 \mathrm{~V}$ to $3.5 \mathrm{~V}$ are applied to the selected nodes on touchpads. A sequential analysis method is chosen to reduce the simulation time, where first the temperature distribution on the device is found by employing temperature and voltage degrees of freedom. While determining the temperature distribution, temperaturedependent heat transfer coefficients given in table 3 are applied as thermal boundary conditions on the surfaces of the device. Then by switching element degrees of freedom to displacements in three dimensions, the deflection profile is obtained through applying the results of the first part as a nodal temperature load on the actuator.

\section{References}

[1] Guckel H, Klein J, Christensen T, Skrobis K, Laudon M and Lovell E G 1992 Thermo-magnetic metal flexure actuators 5th Technical Digest, IEEE Solid-State Sensor and Actuator Workshop (Hilton Head Island, SC, USA, 22-25 June 1992) pp 73

[2] Riethmüller W and Benecke W 1988 Thermally excited silicon microactuators IEEE Trans. Electron. Devices 35758

[3] Mayyas M, Zhang P, Lee W H, Shiakolas P and Popa D 2007 Design tradeoffs for electrothermal microgrippers Proc. IEEE Int. Conf. Robotics Automation (Roma, Italy, April 2007) pp 907

[4] Eichhorn V, Fatikow S, Wich T, Dahmen C, Sievers T, Nordström Andersen K, Carlson K and Bøggild P 2008 Depth-detection methods for microgripper based CNT manipulation in a scanning electron microscope J. Micro-Nano Mech. 427

[5] Liu Z, Wei Y, Chan H-Y, Li W J, Dong Z and Wang Y 2004 Structural and thermal analysis of a thermally actuated polymer micro robotic gripper Proc. IEEE Int. Conf. Robotics and Biomimetics (Shenyang, China, August 2004) pp 470

[6] Incropera F P and DeWitt D P 2002 Fundamentals of Heat and Mass Transfer (New York: Wiley)

[7] ANSYS 10 User Guide

[8] Kakac S and Yener Y 1993 Heat Conduction (Washington, DC: Taylor and Francis)

[9] Guo Z Y and Li Z X 2003 Size effect on single-phase channel flow and heat transfer at microscale Int. J. Heat Fluid Flow 24284

[10] Baglio S, Castorina L, Fortuna L and Savalli N 2002 Modeling and design of novel photo-thermo-mechanical microactuators Sensors Actuators A 101185

[11] Mankame N D and Ananthasuresh G K 2001 Comprehensive thermal modelling and characterization of an electro-thermal-compliant microactuator J. Micromech. Microeng. 11452

[12] Mills A F 1999 Basic Heat and Mass Transfer (Upper Saddle River, NJ: Prentice-Hall)

[13] Jungen A, Pfenninger M, Tonteling M, Stampfer $C$ and Hierold C 2006 Electrothermal effects at the microscale and their consequences on system design J. Micromech. Microeng. 161633

[14] Chen R S, Kung C and Lee G B 2002 Analysis of the optimal dimension on the electrothermal microactuator J. Micromech. Microeng. 12291

[15] Todd S T and Xie H 2005 Steady-state 1D electrothermal modeling of an electrothermal transducer J. Micromech. Microeng. 152264

[16] Yang Y J and Yu C C 2004 Extraction of heat-transfer macromodels for MEMS devices J. Micromech. Microeng. 14587

[17] Milanovic V, Bowen E, Zaghloul M E, Tea N H, Suehle J S, Payne B and Gaitan M 2000 Micromachined convective 
accelerometers in standard integrated circuits technology Appl. Phys. Lett. 76508

[18] Luo X B, Yang Y J, Zheng F, Li Z X and Guo Z Y 2001 An optimized micromachined convective accelerometer with no proof mass J. Micromech. Microeng. 11504

[19] Hickey R, Kujath M and Hubbard T 2002 Heat transfer analysis and optimization of two-beam microelectromechanical thermal actuators $J$. Vac. Sci. Technol. A 20971

[20] Maloney J M, Schreiber D S and DeVoe D L 2004 Large-force electrothermal linear micromotors J. Micromech. Microeng. 14226

[21] Lott C D, McLain T W, Harb J N and Howell L L 2002 Modeling the thermal behavior of a surface-micromachined linear-displacement thermomechanical microactuator Sensors Actuators A 101239

[22] Huang Q A and Lee N K S 1999 Analysis and design of polysilicon thermal flexure actuator J. Micromech. Microeng. 964

[23] Serrano J R, Phinney L M and Kearney S P 2006 Micro-Raman thermometry of thermal flexure actuators J. Micromech. Microeng. 161128

[24] Li L and Uttamchandani D 2004 Modified asymmetric micro-electrothermal actuator: analysis and experimentation J. Micromech. Microeng. 141734
[25] Yan D, Khajepour A and Mansour R 2003 Modeling of two-hot-arm horizontal thermal actuator J. Micromech. Microeng. 13312

[26] Geisberger A A, Sarkar N, Ellis M and Skidmore G D 2003 Electrothermal properties and modeling of polysilicon microthermal actuators J. Microelectromech. Syst. 12513

[27] Kuang Y, Huang Q A and Lee N K S 2002 Numerical simulation of a polysilicon thermal flexure actuator Microsyst. Technol. 817

[28] Zhang Y, Huang Q A, Li R G and Li W 2006 Macro-modeling for polysilicon cascaded bent beam electrothermal microactuators Sensors Actuators A 128165

[29] Li R G, Huang Q A and Li W H 2008 A nodal analysis method for simulating the behavior of electrothermal microactuators Microsyst. Technol. 14119

[30] Huang Q A, Xu G, Qi L and Li W 2006 A simple method for measuring the thermal diffusivity of surface-micromachined polysilicon thin films J. Micromech. Microeng. 16981

[31] Atre A 2006 Analysis of out-of-plane thermal microactuators J. Micromech. Microeng. 16205

[32] Solano B, Rolt S and Wood D 2008 Thermal and mechanical analysis of an SU8 polymeric actuator using infrared thermography Proc. I. Mech. E. Part C: J. Mech. Eng. Sci. 22273

[33] Feynman R P 1992 There's plenty of room at the bottom J. Microelectromech. Syst. 160 